\title{
TRACES OF LOCALISATION OPERATORS WITH TWO ADMISSIBLE WAVELETS
}

\author{
M. W. WONG ${ }^{1}$ and ZHAOHUI ZHANG ${ }^{1}$
}

(Received 12 January, 2001; revised 20 November, 2001)

\begin{abstract}
The resolution of the identity formula for a localisation operator with two admissible wavelets on a separable and complex Hilbert space is given and the traces of these operators are computed.
\end{abstract}

\section{Introduction}

A localisation operator with one admissible wavelet is like the windowed Fourier transform used by Gabor [4] in time-frequency analysis. The admissible wavelet plays the same role in the localisation operator that the window plays in the windowed Fourier transform. We introduce in this paper localisation operators with two admissible wavelets, which can be thought of as windowed Fourier transforms with two windows and hence are better, or more flexible, tools for signal analysis. It turns out that localisation operators with two admissible wavelets have a richer mathematical structure than the one-wavelet analogues, which have been studied in detail in $[3,6,9]$. The natural setting for localisation operators is by now quite well understood to be the theory of square-integrable representations of locally compact and Hausdorff groups on infinite-dimensional, separable and complex Hilbert spaces.

Let $G$ be a locally compact and Hausdorff group on which the left Haar measure is denoted by $\mu$. Let $X$ be a separable and complex Hilbert space, the dimension of which is infinite. We denote the inner product and the norm in $X$ by $($,$) and \|\|$ respectively. Let $B(X)$ be the $C^{*}$-algebra of all bounded linear operators on $X$ and let \|\|$_{*}$ denote the norm in $B(X)$. An irreducible and unitary representation $\pi: G \rightarrow B(X)$ of $G$ on

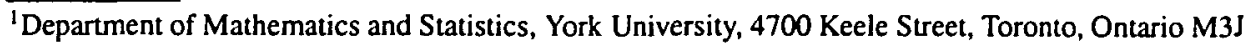
1P3, Canada; e-mail: mwwong@pascal.math.yorku.ca.

(C) Australian Mathematical Society 2003, Serial-fee code 1446-181 1/03
} 
$X$ is said to be square-integrable if there exists a nonzero element $\varphi$ in $X$ such that

$$
\int_{G}|(\varphi, \pi(g) \varphi)|^{2} d \mu(g)<\infty .
$$

We call any element $\varphi$ in $X$ for which $\|\varphi\|=1$ and (1.1) is valid an admissible wavelet for the square-integrable representation $\pi: G \rightarrow B(X)$ and we define the constant $c_{\varphi}$ by $c_{\varphi}=\int_{G}|(\varphi, \pi(g) \varphi)|^{2} d \mu(g)$.

REMARK 1.1. Let $U=\{(b, a): b \in \mathbb{R}, a>0\}$ be the affine group on which the binary operation - is given by $\left(b_{1}, a_{1}\right) \cdot\left(b_{2}, a_{2}\right)=\left(b_{1}+a_{1} b_{2}, a_{1} a_{2}\right)$ for all $\left(b_{1}, a_{1}\right)$ and $\left(b_{2}, a_{2}\right)$ in $U$. Let $H_{+}^{2}(\mathbb{R})$ be the Hardy space defined by

$$
H_{+}^{2}(\mathbb{R})=\left\{f \in L^{2}(\mathbb{R}): \hat{f}=0 \text { a.e. on }(-\infty, 0)\right\},
$$

where $\hat{f}$ is the Fourier transform of $f$. Let $\pi: U \rightarrow H_{+}^{2}(\mathbb{R})$ be the square-integrable representation of $U$ on $H_{+}^{2}(\mathbb{R})$ defined by

$$
(\pi(b, a) f)(x)=\frac{1}{\sqrt{a}} f\left(\frac{x-b}{a}\right), \quad x \in \mathbb{R},
$$

for all $(b, a)$ in $U$ and all $f$ in $H_{+}^{2}(\mathbb{R})$. Then $(1.1)$ is equivalent to the admissibility condition to the effect that

$$
\int_{0}^{\infty} \frac{|\hat{\varphi}(\xi)|^{2}}{\xi} d \xi<\infty
$$

Admissible wavelets $\varphi$ for the square-integrable representation $\pi: U \rightarrow B\left(H_{+}^{2}(\mathbb{R})\right)$ are then exactly the same as the mother wavelets, which have been extensively studied in the literature. See, for instance, [2] for an authoritative account of the subject.

THEOREM 1.2. Let $\varphi$ be an admissible wavelet for the square-integrable representation $\pi: G \rightarrow B(X)$. Then

$$
(x, y)=\frac{1}{c_{\varphi}} \int_{G}(x, \pi(g) \varphi)(\pi(g) \varphi, y) d \mu(g)
$$

for all $x$ and $y$ in $X$.

REMARK 1.3. The formula (1.2) is known as the resolution of the identity formula. Theorem 1.2 is a simplified version of Theorem 3.1 in [5] by Grossmann, Morlet and Paul. A proof can also be found in [9, Chapter 1]. 
Let $F \in L^{\prime}(G)$. Then for any $x$ in $X$, we define $L_{F . \varphi} x$ to be the element in $X$ such that

$$
\left(L_{F, \varphi} x, y\right)=\frac{1}{c_{\varphi}} \int_{G} F(g)(x, \pi(g) \varphi)(\pi(g) \varphi, y) d \mu(g)
$$

for all $y$ in $X$. Then we have the following proposition, which is Proposition 2.1 in [6] by He and Wong. See also [9, Proposition 2.1].

PROPOSITION 1.4. $L_{F, \varphi}: X \rightarrow X$ is a bounded linear operator and

$$
\left\|L_{F, \varphi}\right\|_{*} \leq \frac{1}{c_{\varphi}}\|F\|_{L^{\prime}(G)} .
$$

REMARK 1.5. The bounded linear operator $L_{F, \varphi}: X \rightarrow X$ is called the localisation operator corresponding to the symbol $F$. The reason for the terminology stems from the simple observation that if the symbol $F$ in $L^{1}(G)$ is replaced by the function $H: G \rightarrow \mathbb{C}$ given by $H(g)=1, g \in G$, then the resolution of the identity formula implies that the localisation operator $L_{H, \varphi}: X \rightarrow X$ is simply the identity operator on $X$. Thus, in general, the symbol $F$ is there to localise on $G$ so as to produce a nontrivial bounded linear operator on $X$ with various applications in the mathematical sciences. Localisation operators defined by (1.3) are generalisations of localisation operators studied by Daubechies [1]. See also [2, Section 2.8] in this connection.

Let $A: X \rightarrow X$ be a compact operator. If we denote by $A^{*}: X \rightarrow X$ the adjoint of $A: X \rightarrow X$, then the linear operator $\left(A^{*} A\right)^{1 / 2}: X \rightarrow X$ is positive and compact. Let $\left\{\varphi_{k}: k=1,2, \ldots\right\}$ be an orthonormal basis for $X$ consisting of eigenvectors of $\left(A^{*} A\right)^{1 / 2}: X \rightarrow X$ and let $s_{k}(A)$ be the eigenvalue of $\left(A^{*} A\right)^{1 / 2}$ : $X \rightarrow X$ corresponding to the eigenvector $\varphi_{k}, k=1,2, \ldots$ We say that the compact operator $A: X \rightarrow X$ is in the trace class $S_{1}$ if $\sum_{k=1}^{\infty} s_{k}(A)<\infty$, and we call $s_{k}(A)$, $k=1,2, \ldots$, the singular values of $A$. It can be shown that $S_{1}$ is a Banach space in which the norm \|\|$_{S_{1}}$ is given by

$$
\|A\|_{s_{1}}=\sum_{k=1}^{\infty} s_{k}(A), \quad A \in S_{1} .
$$

The following result, which is well-known, will be useful to us and can be found in $[8$, Section VI.6].

PRoposition 1.6. Let $A: X \rightarrow X$ be a bounded linear operator such that

$$
\sum_{k=1}^{\infty}\left|\left(A \varphi_{k}, \varphi_{k}\right)\right|<\infty
$$

for all orthonormal bases $\left\{\varphi_{k}: k=1,2, \ldots\right\}$ for $X$. Then $A: X \rightarrow X$ is in $S_{1}$. 
If $A: X \rightarrow X$ is a linear operator in $S_{1}$ and $\left\{\varphi_{k}: k=1,2, \ldots\right\}$ is any orthonormal basis for $X$, then it can be proved that the series $\sum_{k=1}^{\infty}\left(A \varphi_{k}, \varphi_{k}\right)$ is absolutely convergent and the sum is independent of the choice of the orthonormal basis $\left\{\varphi_{k}: k=1,2, \ldots\right\}$. Thus we can define the trace $\operatorname{tr}(A)$ of $A: X \rightarrow X$ by $\operatorname{tr}(A)=\sum_{k=1}^{\infty}\left(A \varphi_{k}, \varphi_{k}\right)$, where $\left\{\varphi_{k}: k=1,2, \ldots\right\}$ is any orthonormal basis for $X$. It is now easy to see that if $A: X \rightarrow X$ is a positive operator in $S_{1}$, then $\|A\|_{S_{1}}=\operatorname{tr}(A)$.

The following result is Theorem 2.2 in [3] by Du and Wong. A proof can also be found in $[9$, Chapter 4$]$.

PROPOSITION 1.7. Let $F \in L^{1}(G)$. Then the localisation operator $L_{F, \varphi}: X \rightarrow X$ is in $S_{1}$ and

$$
\operatorname{tr}\left(L_{F, \varphi}\right)=\frac{1}{c_{\varphi}} \int_{G} F(g) d \mu(g) .
$$

The results hitherto described are for localisation operators $L_{F, \varphi}: X \rightarrow X$ defined in terms of one admissible wavelet $\varphi$ for the square-integrable representation $\pi: G \rightarrow$ $B(X)$ of $G$ on $X$. In this paper, we introduce the notion of a localisation operator $L_{F, \varphi, \psi}: X \rightarrow X$, which is defined in terms of a symbol $F$ in $L^{1}(G)$ and two admissible wavelets $\varphi$ and $\psi$ for the square-integrable representation $\pi: G \rightarrow B(X)$ of $G$ on $X$. It is proved in Section 3 that $L_{F, \varphi, \psi}: X \rightarrow X$ is in $S_{1}$ and a formula for the trace of $L_{F, \varphi, \psi}: X \rightarrow X$ is given. These results extend, respectively, the corresponding results in $[6,3]$ from the one-wavelet case to the two-wavelet case. The main tool that we need is an analogue of the resolution of the identity formula (1.2) for two admissible wavelets for the square-integrable representation $\pi: G \rightarrow B(X)$ of $G$ on $X$, and this is given in Section 2.

The motivation for the trace formula comes from the study of the free energy and the partition function in quantum statistical mechanics as described in, for example, [7].

\section{A resolution of the identity formula}

In this section, we give an analogue of the resolution of the identity formula (1.2) for two admissible wavelets for the square-integrable representation $\pi: G \rightarrow B(X)$ of $G$ on $X$.

THEOREM 2.1. Let $\varphi$ and $\psi$ be two admissible wavelets for the square-integrable representation $\pi: G \rightarrow B(X)$ of $G$ on $X$. Then

$$
\int_{G}(x, \pi(g) \varphi)(\pi(g) \psi, y) d \mu(g)=c_{\varphi, \psi}(x, y), \quad x, y \in X,
$$


where

$$
c_{\varphi, \psi}=\int_{G}(\varphi, \pi(g) \varphi)(\pi(g) \psi, \varphi) d \mu(g) .
$$

Proof. First we define $A_{\varphi}: X \rightarrow L^{2}(G)$ and $A_{\psi}: X \rightarrow L^{2}(G)$, respectively, by

$$
\left(A_{\varphi} x\right)(g)=(x, \pi(g) \varphi), \quad x \in X, g \in G,
$$

and

$$
\left(A_{\psi} x\right)(g)=(x, \pi(g) \psi), \quad x \in X, g \in G
$$

Then, by (1.2), $A_{\varphi}: X \rightarrow L^{2}(G)$ and $A_{\psi}: X \rightarrow L^{2}(G)$ are bounded linear operators. By (2.3) and the fact that $\pi: G \rightarrow B(X)$ is a unitary representation, we get, for $x \in X$, and $g, h \in G$,

$$
\left(A_{\varphi} \pi(h) x\right)(g)=(\pi(h) x, \pi(g) \varphi)=\left(x, \pi\left(h^{-1} g\right) \varphi\right)=\left(A_{\varphi} x\right)\left(h^{-1} g\right) .
$$

So

$$
A_{\varphi} \pi(h)=L(h) A_{\varphi}
$$

where

$$
(L(h) f)(g)=f\left(h^{-1} g\right), \quad g, h \in G,
$$

for all $f$ in $L^{2}(G)$. Now, for all $x$ and $y$ in $X$, we get, by (2.4)-(2.7) and the fact that $\pi: G \rightarrow B(X)$ is a unitary representation,

$$
\begin{aligned}
\left(A_{\psi}^{*} L(h) A_{\varphi} x, y\right) & =\left(L(h) A_{\varphi} x, A_{\psi} y\right)_{L^{2}(G)}=\int_{G}\left(A_{\varphi} x\right)\left(h^{-1} g\right) \overline{\left(A_{\psi} y\right)(g)} d \mu(g) \\
& =\int_{G}\left(A_{\varphi} x\right)(g) \overline{\left(A_{\psi} y\right)(h g)} d \mu(g)=\left(A_{\varphi} x, L\left(h^{-1}\right) A_{\psi} y\right)_{L^{2}(G)} \\
& =\left(A_{\varphi} x, A_{\psi} \pi\left(h^{-1}\right) y\right)_{L^{2}(G)}=\left(\pi(h) A_{\psi}^{*} A_{\varphi} x, y\right), \quad h \in G,
\end{aligned}
$$

where $(,)_{L^{2}(G)}$ is the inner product in $L^{2}(G)$. Thus, by (2.8), we get

$$
A_{\psi}^{*} L(h) A_{\varphi}=\pi(h) A_{\psi}^{*} A_{\varphi}, \quad h \in G
$$

Hence, by (2.6) and (2.9), we get

$$
A_{\psi}^{*} A_{\varphi} \pi(h)=\pi(h) A_{\psi}^{*} A_{\varphi}, \quad h \in G .
$$

From (2.10) and the fact that $\pi: G \rightarrow B(X)$ is an irreducible representation, we conclude by Schur's lemma that there exists a constant $c_{\varphi, \psi}$ such that

$$
A_{\psi}^{*} A_{\varphi}=c_{\varphi, \psi} I
$$


where $I$ is the identity operator on $X$. So, by (2.11), we get

$$
\begin{aligned}
c_{\varphi, \psi}(x, y) & =\left(c_{\varphi, \psi} I x, y\right)=\left(A_{\psi}^{*} A_{\varphi} x, y\right)=\left(A_{\varphi} x, A_{\psi} y\right)_{L^{2}(G)} \\
& =\int_{G}(x, \pi(g) \varphi) \overline{(y, \pi(g) \psi)} d \mu(g) \\
& =\int_{G}(x, \pi(g) \varphi)(\pi(g) \psi, y) d \mu(g), \quad x, y \in X,
\end{aligned}
$$

and the proof is complete if we let $x=y=\varphi$ and note that $\|\varphi\|=1$ in (2.12).

REMARK 2.2. If $c_{\varphi, \psi} \neq 0$, then by $(2.1)$, we have

$$
(x, y)=\frac{1}{c_{\varphi, \psi}} \int_{G}(x, \pi(g) \varphi)(\pi(g) \psi, y) d \mu(g), \quad x, y \in X,
$$

and we call this formula the resolution of the identity formula for the square-integrable representation $\pi: G \rightarrow B(X)$ of $G$ on $X$ corresponding to the admissible wavelets $\varphi$ and $\psi$.

REMARK 2.3. It is proved in [5, p. 2475] by Grossmann, Morlet and Paul that

$$
\int_{G}(x, \pi(g) \varphi)(\pi(g) \psi, y) d \mu(g)=(A \varphi, A \psi)(x, y), \quad x, y \in X,
$$

where $A$ is a positive and self-adjoint operator on $X$. Thus, by Theorem 2.1 , we get $c_{\varphi, \psi}=(A \varphi, A \psi)$ for all admissible wavelets $\varphi$ and $\psi$ for the square-integrable representation $\pi: G \rightarrow B(X)$ of $G$ on $X$. If we define $\langle\varphi, \psi\rangle$ for all $\varphi$ and $\psi$ in the set $A W$ of all admissible wavelets for the square-integrable representation $\pi: G \rightarrow B(X)$ of $G$ on $X$ by $\langle\varphi, \psi\rangle=c_{\varphi, \psi}$, then $\langle$,$\rangle is an inner product in A W$. It should be pointed out that the proof of the fact that $\langle$,$\rangle is an inner product in A W$ is an easy consequence of (2.2), and does not depend on the more technical formula (2.13), which is proved in [5].

REMARK 2.4. That the inner product $\langle$,$\rangle in A W$ is just the inner product (, ) in $X$ for unimodular groups is the content of the following theorem, which also tells us when $c_{\varphi, \psi} \neq 0$ for unimodular groups. Although the theorem is an immediate consequence of Theorem 3.1 (iii) and Equation (3.2) in [5], the simple and direct proof that we give in this paper is of some interest in its own right.

THEOREM 2.5. Let $G$ be a unimodular group, and let $\varphi$ and $\psi$ be two admissible wavelets for the square-integrable representation $\pi: G \rightarrow B(X)$ of $G$ on $X$. Then

$$
c_{\varphi, \psi}=(\psi, \varphi) c_{\varphi}
$$


Proof. By (2.2) and the fact that $\pi: G \rightarrow B(X)$ is a unitary representation,

$$
\begin{aligned}
c_{\varphi, \psi} & =\int_{G}\left(\psi, \pi\left(g^{-1}\right) \varphi\right)\left(\pi\left(g^{-1}\right) \varphi, \varphi\right) d \mu(g) \\
& =\int_{G}(\psi, \pi(g) \varphi)(\pi(g) \varphi, \varphi) d \tilde{\mu}(g),
\end{aligned}
$$

where $\tilde{\mu}(B)=\mu\left(B^{-1}\right)$ for all measurable subsets $B$ of $G$, and $B^{-1}=\left\{g^{-1}: g \in B\right\}$. Now, using the unimodularity of $G$,

$$
\mu=\tilde{\mu} .
$$

So, by (1.2), (2.15) and (2.16), we get (2.14).

The following consequence of formula (2.2) for $c_{\varphi, \psi}$ and Theorem 2.5 holds for unimodular groups, and is an interesting result in its own right.

THEOREM 2.6. Let $G$ be a unimodular group, and let $\varphi$ and $\psi$ be two admissible wavelets for the square-integrable representation $\pi: G \rightarrow B(X)$ of $G$ on $X$. Then $c_{\varphi}=c_{\psi}$.

Proof. Putting $x=y=\psi$ in (2.1), we get

$$
c_{\varphi, \psi}=\int_{G}(\psi, \pi(g) \varphi)(\pi(g) \psi, \psi) d \mu(g)
$$

By (2.2) and (2.17), we get

$$
c_{\psi, \varphi}=\overline{c_{\varphi, \psi}} .
$$

So, by Theorem $2.5,(2.18)$ and the fact that $c_{\varphi}$ is real-valued,

$$
(\varphi, \psi) c_{\psi}=\overline{(\psi, \varphi)} c_{\varphi}=(\varphi, \psi) c_{\varphi}
$$

So $c_{\varphi}=c_{\psi}$ if $(\varphi, \psi) \neq 0$. Now, suppose that $(\varphi, \psi)=0$. Let $\omega \in X$ be such that $\|\omega\|=1,(\omega, \varphi) \neq 0$ and $(\omega, \psi) \neq 0$. As has been shown in [5], $\omega$ is an admissible wavelet for the square-integrable representation $\pi: G \rightarrow B(X)$. Thus, by what we have just shown, $c_{\varphi}=c_{\omega}$ and $c_{\psi}=c_{\omega}$. Hence $c_{\varphi}=c_{\psi}$ and the proof is complete.

\section{The main result}

Let $\varphi$ and $\psi$ be admissible wavelets for the square-integrable representation $\pi$ : $G \rightarrow B(X)$ of $G$ on $X$ such that $c_{\varphi, \psi} \neq 0$, where $c_{\varphi . \psi}$ is given by (2.2). Let $F \in L^{1}(G)$. Then we define the localisation operator $L_{F, \varphi, \psi}: X \rightarrow X$ by

$$
\left(L_{F, \varphi, \psi} x, y\right)=\frac{1}{c_{\varphi, \psi}} \int_{G} F(g)(x, \pi(g) \varphi)(\pi(g) \psi, y) d \mu(g), \quad x, y \in X .
$$


THEOREM 3.1. The localisation operator $L_{F, \varphi, \psi}: X \rightarrow X$ is in $S_{1}$ and

$$
\operatorname{tr}\left(L_{F, \varphi, \psi}\right)=\frac{(\psi, \varphi)}{c_{\varphi, \psi}} \int_{G} F(g) d \mu(g) .
$$

Proof. By (3.1), the Schwarz inequality, the fact that $\pi: G \rightarrow B(X)$ is a unitary representation, and $\|\varphi\|=\|\psi\|=1$, we get

$$
\begin{aligned}
\left|\left(L_{F, \varphi, \psi} x, y\right)\right| & \leq \frac{1}{\left|c_{\varphi, \psi}\right|} \int_{G}|F(g)|\|\pi(g) \varphi\|\|\pi(g) \psi\| d \mu(g)\|x\|\|y\| \\
& =\frac{1}{\left|c_{\varphi, \psi}\right|}\|F\|_{L^{\prime}(G)}\|x\|\|y\|, \quad x, y \in X .
\end{aligned}
$$

Thus $L_{F, \varphi, \psi}: X \rightarrow X$ is a bounded linear operator. Next, let $\left\{\varphi_{k}: k=1,2, \ldots\right\}$ be an orthonormal basis for $X$. Then, by Fubini's theorem, Schwarz' inequality, Parseval's identity, the fact that $\pi: G \rightarrow B(X)$ is a unitary representation, and $\|\varphi\|=\|\psi\|=1$, we get

$$
\begin{aligned}
\sum_{k=1}^{\infty} \mid & \left(L_{F, \varphi, \psi} \varphi_{k}, \varphi_{k}\right) \mid \\
& \leq \frac{1}{\left|c_{\varphi, \psi}\right|} \sum_{k=1}^{\infty} \int_{G}|F(g)|\left|\left(\varphi_{k}, \pi(g) \varphi\right)\right|\left|\left(\pi(g) \psi, \varphi_{k}\right)\right| d \mu(g) \\
& \leq \frac{1}{\left|c_{\varphi, \psi}\right|} \int_{G}|F(g)|\left\{\sum_{k=1}^{\infty}\left|\left(\varphi_{k}, \pi(g) \varphi\right)\right|^{2}\right\}^{1 / 2}\left\{\sum_{k=1}^{\infty}\left|\left(\pi(g) \psi, \varphi_{k}\right)\right|^{2}\right\}^{1 / 2} d \mu(g) \\
& =\frac{1}{\left|c_{\varphi, \psi}\right|} \int_{G}|F(g)| \|(\pi(g) \varphi\|\| \pi(g) \psi \| d \mu(g) \\
& =\frac{1}{\left|c_{\varphi, \psi}\right|}\|F\|_{L^{1}(G)}<\infty
\end{aligned}
$$

So, by (3.2) and Proposition 1.7, $L_{F, \varphi, \psi}: X \rightarrow X$ is in $S_{1}$. Finally, let $\left\{\varphi_{k}: k=\right.$ $1,2, \ldots\}$ be any orthonormal basis for $X$. Then, by Fubini's theorem, Parseval's identity, the fact that $\pi: G \rightarrow B(X)$ is a unitary representation, and $\|\varphi\|=\|\psi\|=1$, we get

$$
\begin{aligned}
\operatorname{tr}\left(L_{F, \varphi, \psi}\right) & =\sum_{k=1}^{\infty}\left(L_{F, \varphi, \psi} \varphi_{k}, \varphi_{k}\right)=\frac{1}{c_{\varphi, \psi}} \sum_{k=1}^{\infty} \int_{G} F(g)\left(\varphi_{k}, \pi(g) \varphi\right)\left(\pi(g) \psi, \varphi_{k}\right) d \mu(g) \\
& =\frac{1}{c_{\varphi, \psi}} \int_{G} F(g) \sum_{k=1}^{\infty}\left(\varphi_{k}, \pi(g) \varphi\right)\left(\pi(g) \psi, \varphi_{k}\right) d \mu(g) \\
& =\frac{1}{c_{\varphi, \psi}} \int_{G} F(g)(\pi(g) \psi, \pi(g) \varphi) d \mu(g)=\frac{(\psi, \varphi)}{c_{\varphi, \psi}} \int_{G} F(g) d \mu(g),
\end{aligned}
$$

and the proof is complete. 


\section{Acknowledgements}

The authors would like to thank the editor and the referees for their constructive comments which have improved the contents and the presentation of the paper.

This research has been partially supported by the Natural Sciences and Engineering Research Council of Canada under Grant OGP0008562.

\section{References}

[1] I. Daubechies, "Time-frequency localization operators: a geometric phase space approach", IEEE Trans. Inform. Theory 34 (1988) 605-612.

[2] I. Daubechies, Ten lectures on wavelets (SIAM, 1992).

[3] J. Du and M. W. Wong, "Traces of localization operators", C. R. Math. Rep. Acad. Sci. Canada 22 (2000) 92-96.

[4] D. Gabor, "Theory of communications", J. Inst. Elec. Eng. (London) 93 (1946) 429-457.

[5] A. Grossmann, J. Morlet and T. Paul, "Transforms associated to square integrable group representations I: general results", J. Math. Phys. 26 (1985) 2473-2479.

[6] Z. He and M. W. Wong, "Localization operators associated to square integrable group representations", Panamer. Math. J. 6 (1996) 93-104.

[7] R. Peierls, "On a minimal property of the free energy", Phys. Rev. 54 (1938) 918-919.

[8] M. Reed and B. Simon, Methods of modern mathematical physics I: functional analysis, revised and enlarged ed. (Academic Press, 1980).

[9] M. W. Wong, Localization operators, Lecture Notes Series 47 (Seoul National University, Research Institute of Mathematics, Global Analysis Research Center, Seoul, 1999). 